\title{
STOCHASTIC NAVIER-STOKES EQUATIONS ON 2D ROTATING SPHERES WITH STABLE LÉVY NOISE
}

\author{
LEANNE DONG
}

(Received 21 October 2018; first published online 7 January 2019)

\begin{abstract}
2010 Mathematics subject classification: primary 60H15; secondary 35Q30, 60H30, 76D03, 76D05, $76 \mathrm{D} 06$.

Keywords and phrases: stochastic Navier-Stokes equations with Lévy noise, $\beta$-stable noise, unit spheres, random dynamical system, invariance measures, random attractor.
\end{abstract}

The Navier-Stokes equations on 2D spheres coupled to thermodynamics are generally accepted as a natural model for global atmospheric circulation on Earth. Stochastic analysis of geophysical flow models driven by Gaussian noise is a relatively young area of research that has made great mathematical advances over the past few years. These models are developed to tackle defence-related problems such as atmospheric and ocean data assimilation. On the other hand, motivated from engineering sciences, the study of stochastic Navier-Stokes equations (SNSE) is moving toward perturbation with Lévy noise.

In this thesis, we study the SNSE on 2D rotating spheres perturbed by pure jump Lévy noise of $\beta$-stable type. The first goal is to establish the well-posedness of solutions to this class of equations. The second goal is to investigate qualitative questions on ergodicity, asymptotic behaviour and random dynamics. In Chapter 2, we review the analytic and probabilistic preliminaries required to present the main results of the thesis. Then we introduce the background material on Hilbert space valued cylindrical Lévy noise via subordination of $\beta$-stable type. In Chapter 3 , we prove the existence and uniqueness of solutions to the SNSE under suitable assumptions of noise and forcing and, in the second part, we deduce the existence of an invariant measure with measure support. Chapter 4 is devoted to the study of random dynamical systems generated by our SNSE. In particular, we prove that, with sufficient regularity, there exists a finite-dimensional random attractor for our SNSE. Moreover, such a random attractor supports a Feller-Markov invariant measure.

Thesis submitted to the University of Sydney in February 2018; degree approved on 23 April 2018; principal supervisor Beniamin Goldys.

(c) 2019 Australian Mathematical Publishing Association Inc. 
LEANNE DONG, School of Mathematics and Statistics, University of Sydney, New South Wales 2006, Australia e-mail: jdleanne@gmail.com 